\title{
L-Ornithine L-Aspartate (LOLA) for the Treatment of Hepatic Encephalopathy in Cirrhosis: Novel Insights and Translation to the Clinic
}

\author{
Roger F. Butterworth ${ }^{1}$
}

Published online: 31 January 2019

๑) Springer Nature Switzerland AG 2019

The principal objective of this dedicated Supplement to the journal focuses on the demystification of the concept of hepatic encephalopathy (HE) in cirrhosis. Particular attention is paid to the definition of HE, its subtypes in relation to the nature and severity of liver impairment, the scales used for assessment of HE grades, and approaches to diagnosis. The effects of HE in cirrhosis on quality-of-life issues for the patients and their families and caregivers are given particular prominence. An update on the pathology and pathobiology of HE in cirrhosis follows in which the roles of exposure of the brain to gut-derived toxins (ammonia, manganese), inflammation and enhanced central inhibitory neurotransmission are described. The following manuscript in the Supplement deals with the pharmacokinetics/pharmacodynamics of L-ornithine L-aspartate (LOLA), following its discovery as an effective ammonia-lowering agent some 50 years ago. Details relating to LOLA's mechanisms of action and a critical review of the evidence from clinical trials and meta-analyses in support of the efficacy of LOLA for the treatment of HE in cirrhosis is included together with preliminary evidence of efficacy in non-alcoholic fatty liver disease (NAFLD).

HE is defined in general terms as brain dysfunction caused by liver insufficiency and/or portal-systemic shunting of venous blood. The lead article of The Supplement by Karin Weissenborn (Hannover, Germany), a clinical neurologist and key opinion leader in the field, describes the spectrum of clinical neurological symptoms characteristic of HE in cirrhosis (currently referred to as Type C HE). The condition is frequently associated with a precipitating factor such as a gastrointestinal bleed, infection or electrolyte imbalance. The most widely used system for grading of

Roger F. Butterworth

rb@enceph.com

1 Department of Medicine, University of Montreal, 45143

Cabot Trail, Englishtown, NS B0C 1H0, Canada overt HE (OHE) in cirrhosis is West Haven criteria (WHC). Patients with liver cirrhosis without clinically overt symptoms of HE but with evidence of CNS dysfunction based upon the results of neuropsychological or neurophysiological testing are considered to have minimal hepatic encephalopathy (MHE). However, in order to address the inherent subjective nature of early HE grades using WHC, it has been suggested that the term MHE be replaced by the term, "covert HE" that encompasses the previous MHE plus grade I HE as defined by WHC. This new nomenclature is largely being adopted. Karin Weissenborn goes on to draw attention to the fact that that persistent neurological symptoms, due largely to focal neurodegenerative pathology, may also occur in Type C HE and these include acquired non-Wilsonian hepatocerebral degeneration, cerebellar degeneration, and extrapyramidal symptoms resulting from a variant of Parkinsonism. Diagnosis of HE is hampered by the fact that none of the symptoms are specific in nature. Consequently, a diagnosis of $\mathrm{HE}$ can be made only after exclusion of other possible brain disorders [1].

HE has significant negative impact on health-related quality of life (HRQOL) in patients with cirrhosis with significant clinical and psychosocial consequences as described in the article by Sara Montagnese (Padova, Italy) and Jasmohan Bajaj (Richmond, USA), two internationally renowned specialists in the field. Methods employed for the assessment of HRQOL in cirrhosis are described that include the use of generic or liver-specific instruments. HRQOL abnormalities may span multiple areas of daily functioning and changes in sleep-wake cycle patterns having significant negative impact upon the patient's ability to find employment or to operate machinery including an automobile. Sleep-wake cycle changes have a major effect on HRQOL that remain challenging to treat. HRQOL in cirrhosis is modulated by multiple factors in addition to the presence and severity of HE, including the etiology of cirrhosis and an element known as cognitive reserve. Patients with higher cognitive reserve 
are able to tolerate the impact of HE on HRQOL better than those with poor cognitive reserve. HRQOL impairment due to HE has a negative impact not only on patients' daily functioning but also on that of their families and caregivers. Caregivers of patients with HE can face substantial financial and psychological burdens that may affect personal health and longevity. The authors make a plea for the regular assessment of HRQOL in this population [2].

The cellular neuropathology of Type C HE described by Roger Butterworth (Montreal, Canada) is primarily astroglial in nature characterized by a characteristic pathological change known as Alzheimer type 2 astrocytosis, together with activation of microglia indicative of the presence of neuroinflammation (inflammation of the brain per se). Focal loss of neurons may also occur in certain basal ganglia, thalamic, and cerebellar structures as mentioned above. The pathophysiology of HE in cirrhosis is multifactorial involving brain accumulation of ammonia and manganese, systemic and central inflammation, nutritional/metabolic factors and a unique activation of the GABAergic neurotransmitter system caused increased brain concentrations of the neurosteroid allopregnanolone, shown to accumulate in brain in patients with cirrhosis and grade 4 HE. Neuroimaging and spectroscopic techniques reveal early deactivation of the anterior cingulate cortex in parallel with deficits in attention in patients with cirrhosis and mild HE. On the other hand, T1-weighted magnetic resonance signal hyperintensities in basal ganglia resulting from manganese deposits lead to the development of a novel clinical entity referred to as Parkinsonism in cirrhosis. Elucidation of pathophysiologic mechanisms started with the notion that primary impairment of brain energy metabolism was the cause of HE in cirrhosis, a concept that has been disproven. Current therapeutic approaches to HE are aimed largely at reduction of blood ammonia by the use of non-absorbable disaccharides, antibiotics or LOLA. Studies are underway to evaluate the efficacy of agents aimed at reduction of systemic and central inflammation or the reduction of GABAergic tone via recently discovered antagonists of the neurosteroid-modulatory site on the GABA receptor complex [3].

Gerald Kircheis and Stefan Lueth (Brandenburg, Germany) provide a timely review of the pharmacokinetics and pharmacodynamics of LOLA. Being a stable salt of L-ornithine and L-aspartate, LOLA readily dissociates into its constituent amino acids that are readily absorbed by active transport, distributed and metabolized with L-ornithine serving as intermediate in the urea cycle in periportal hepatocytes of the liver and by transamination of L-ornithine to glutamate the substrate for glutamine synthetase located in perivenous hepatocytes as well as in skeletal muscle and brain. The nature, cellular and biological location of these metabolic pathways underpins the rationale for the use of LOLA as an effective ammonia-lowering strategy widely used for the management and treatment of HE in cirrhosis. More recent studies suggest that LOLA may have direct hepatoprotective actions [4].

The article by Mark McPhail (London, UK) and Roger Butterworth (Montreal, Canada) conducts an appraisal of the evidence in support of LOLA for the management and treatment of HE in cirrhosis. Meta-analyses of randomized controlled trials (RCTs) conducted over the last two decades provide evidence of benefit of LOLA in a range of clinical HE presentations including improvement of mental state grade in OHE assessed by WHC as well as in MHE assessed by psychometric testing where the oral formulation of LOLA was found to be particularly effective. Concerns over study quality were noted in one metaanalysis. The effective lowering of fasting blood ammonia was reported in all published RCTs using this endpoint. Network meta-analyses showed that LOLA is comparable (or superior) in efficacy to non-absorbable disaccharides or probiotics. Emerging evidence from RCTs shows efficacy of LOLA for the treatment of post-TIPSS HE as well as for primary and secondary prophylaxis. If confirmed in larger analyses, these findings could provide a considerable basis of support for the use of LOLA in the prevention of HE in cirrhosis [5].

In the final article of The Supplement, Ali Canbay and Jan-Peter Sowa (Magdeburg, Germany) raise the point that, while LOLA has been successfully applied for the treatment of HE and hyperammonemia in cirrhosis, it is less clear whether LOLA could be used in NAFLD. NAFLD, and the progressive form non-alcoholic steatohepatitis (NASH), are currently the leading causes of chronic liver disease worldwide, with roughly $25 \%$ of the world population affected by NAFLD. Consequences of NASH are end-stage liver disease and cardiovascular morbidity and mortality. As the basis for NAFLD is excess calorie uptake and excess adipose tissue mass, the conservative therapeutic approach is weight loss by intense lifestyle change. However, no pharmacological treatment options are currently approved. LOLA is being investigated as a pharmacological tool to ameliorate liver injury in NAFLD on the basis that it lowers liver ammonia concentrations and stimulates the production of antioxidants [6].

\section{Compliance with Ethical Standards}

Funding and Conflicts of interest Funding for studies in the author's research unit was provided by The Canadian Institutes for Health Research. This supplement to Drugs has been wholly funded by Merz Pharmaceuticals GmbH, Frankfurt, Germany. 


\section{References}

1. Weissenborn K. Hepatic encephalopathy: definition, clinical grading and diagnostic principles. Drugs. 2018. https://doi. org/10.1007/s40265-018-1018-z.

2. Montagnese S, Bajaj JS. Impact of hepatic encephalopathy in cirrhosis on quality of life issues. Drugs. 2018. https://doi. org/10.1007/s40265-018-1019-y.

3. Butterworth RF. Hepatic encephalopathy in cirrhosis: pathology and pathophysiology. Drugs. 2018. https://doi.org/10.1007/s4026 5-018-1017-0.
4. Kircheis G, Lüth S. Pharmacokinetic and pharmacodynamic properties of L-ornithine L-aspartate (LOLA) in Hepatic Encephalopathy (HE). Drugs. 2018. https://doi.org/10.1007/s4026 5-018-1023-2.

5. Butterworth RF, McPhail MJW. L-ornithine L-aspartate for hepatic encephalopathy in cirrhosis: results of randomized controlled trials and meta-analyses. Drugs. 2018. https://doi.org/10.1007/s4026 5-018-1024-1.

6. Canbay A, Sowa JP. L-ornithine L-aspartate as novel approach for therapy of non-alcoholic fatty liver disease. Drugs. 2018. https:// doi.org/10.1007/s40265-018-1020-5. 